\title{
Gonadotropin-Dependent Precocious Puberty
}

National Cancer Institute

\section{Source}

National Cancer Institute. Gonadotropin-Dependent Precocious Puberty. NCI Thesaurus.

Code C113217.

Premature onset of sexual development triggered by the premature secretion of gonadotropins. 